\title{
Selection of drug resistant mutants from random library of Plasmodium falciparum dihydrofolate reductase in Plasmodium berghei model
}

\author{
Wachiraporn Tipsuwan ${ }^{1,2}$, Somdet Srichairatanakool ${ }^{2}$, Sumalee Kamchonwongpaisan ${ }^{1}$, Yongyuth Yuthavong ${ }^{1}$ and \\ Chairat Uthaipibull ${ }^{*}$
}

\begin{abstract}
Background: The prevalence of drug resistance amongst the human malaria Plasmodium species has most commonly been associated with genomic mutation within the parasites. This phenomenon necessitates evolutionary predictive studies of possible resistance mutations, which may occur when a new drug is introduced. Therefore, identification of possible new Plasmodium falciparum dihydrofolate reductase (PFDHFR) mutants that confer resistance to antifolate drugs is essential in the process of antifolate anti-malarial drug development.

Methods: A system to identify mutations in Pfdhfr gene that confer antifolate drug resistance using an animal Plasmodium parasite model was developed. By using error-prone PCR and Plasmodium transfection technologies, libraries of Pfdhfr mutant were generated and then episomally transfected to Plasmodium berghei parasites, from which pyrimethamine-resistant PfDHFR mutants were selected.

Results: The principal mutation found from this experiment was $\$ 108 \mathrm{~N}$, coincident with the first pyrimethamineresistance mutation isolated from the field. A transgenic $P$. berghei, in which endogenous Pbdhfr allele was replaced with the mutant Pfdhfr ${ }^{508 \mathrm{~N}}$, was generated and confirmed to have normal growth rate comparing to parental nontransgenic parasite and also confer resistance to pyrimethamine.

Conclusion: This study demonstrated the power of the transgenic $P$. berghei system to predict drug-resistant Pfdhfr mutations in an in vivo parasite/host setting. The system could be utilized for identification of possible novel drugresistant mutants that could arise against new antifolate compounds and for prediction the evolution of resistance mutations.
\end{abstract}

\section{Background}

Plasmodium falciparum, the most virulent malaria pathogen species, is responsible for nearly 863,000 deaths in 2009 [1]. Malaria treatment is hampered by existence of only a limited number of drugs and the emergence of parasites resistant to most available antimalarial drugs, including chloroquine, pyrimethamine and proguanil. Therefore, there is an urgent need to search for affordable, effective and safe anti-malarials that can combat drug resistant parasites.

\footnotetext{
* Correspondence: chairat@biotec.or.th

'National Center for Genetic Engineering and Biotechnology (BIOTEC), National Science and Technology Development Agency (NSTDA), 113

Thailand Science Park, Pathumthani 12120, Thailand

Full list of author information is available at the end of the article
}

A bifunctional enzyme in the folate biosynthesis pathway, dihydrofolate reductase-thymidylate synthase (DHFR-TS) is a well-defined target of traditional antimalarial drugs such as pyrimethamine and cycloguanil [2]. DHFR catalyses the production of tetrahydrofolate from dihydrofolate while TS is in charge of transferring a methyl-group from N5, N10-methylene-tetrahydrofolate to dUMP thereby generating dTMP and tetrahydrofolate. Mutations in Pfdhfr gene, associated with the amino acid substitution at residues $51,59,108$, and 164 , have been found in the field with different levels of resistance to antifolate drugs $[3,4]$. These mutations are positioned around the enzyme active site. Thus, the accumulation of point mutations in Pfdhfr reduces the affinity of antifolate drugs such as pyrimethamine for the enzyme leading to 
drug resistance [5,6]. These correlations of drug resistance with mutations in Pfdhfr gene have been experimentally verified by comparison of mutant enzymes in transfected parasites [7]. Despite the emergence of resistant Pfdhfr mutants in malaria endemic areas, the elucidation and development of target based screening models $[8,9]$ and the solution of the crystal structure of PfDHFRTS [10] means that this enzyme is still an attractive target for drug development. Therefore, in order to develop new compounds against drug resistant parasites, prediction of possible future resistance mutations becomes a priority.

Studies of mutant PfDHFR-TS expression in nonPlasmodium surrogate systems, such as Escherichia coli and Saccharomyces cerevisiae have successfully identified novel mutant Pfdhfr alleles that confer antifolate drug resistance $[8,11]$. These systems have also been useful in testing new compounds against the enzyme $[8,9]$. However, the biochemical activities of some mutant enzymes isolated using these systems have been shown to be significantly lower than the wild-type enzyme [11]. The physiology of these surrogate cells are markedly different from Plasmodium parasites, and it is not clear whether these resistant enzymes would have sufficient enzymatic activity to support parasite growth throughout all stages of the life cycle. Unfortunately owing to the narrow primate host range, suitable Plasmodium in vivo models are not available.

Rodent malaria parasites such as Plasmodium berghei are attractive models for human malaria, and the recent advances in DNA transfection technology allow for genetic modification of the parasite $[12,13]$. DNA transfection can be used to express $P$. falciparum genes in $P$. berghei, and thus transgenic $P$. berghei parasites can be used as in vivo surrogate models. In this study, the power of the transgenic $P$. berghei system to predict drug-resistant Pfdhfr mutations in an in vivo parasite/ host setting was demonstrated. The objective was to identify mutations in Pfdhfr that confer resistance to anti-folate drugs using a transgenic $P$. berghei model. Libraries of randomly mutated Pfdhfr were generated by PCR mutagenesis and transfected to P. berghei. Upon transfection, resistant parasites were obtained after pyrimethamine selection and found to harbor Pfdhfr mutant alleles. Thus, this system can be used as a Plasmodium surrogate system for more accurate prediction and identification of antifolate-resistance mutations.

\section{Methods}

\section{Experimental animals and parasite}

For all experiments, female BALB/c mice (National Laboratory Animal Center, Mahidol University, Thailand) 4-6 weeks old and weighing 20-25 g were used for $P$. berghei parasite infections. The transgenic $P$. berghei parasite line MRA-867 expressing green fluorescent protein without drug-resistant selectable marker (PbGFP), kindly provided by Drs. Andrew Waters and Chris Janse of Malaria Research Group, Leiden University Medical Center, the Netherlands, was used in this study [14]. All animal work was evaluated and approved by the Ethical Committee on Animal Experimentation, National Center for Genetic Engineering and Biotechnology (BIOTEC), Thailand, and followed international guidelines for the use of animals in experimental studies.

\section{Construction of $P$. berghei transfection plasmid}

Plasmid for $P$. berghei transfection in this study was modified from the original plasmid pL0017 [15], which was kindly provided by Drs. Andrew Waters and Chris Janse (Leiden University Medical Centre, the Netherlands). The final transfection plasmid, designated pY005, contains wild type Pfdhfr-ts gene flanked with 1.0-kb each of 5' and 3' untranslated region (UTR) sequences of Pbdhfr-ts. BamHI and AflII restriction sites were introduced at 5' and 3' ends, respectively, of $P f d h f r$ domain to serve as cloning sites for the randomly-mutated Pfdhfr library.

\section{Construction of Pfdhfr random mutant library}

Pfdhfr mutant library was generated by error prone PCR [11]. The $50 \mu \mathrm{L}$ PCR reaction contained $1 \mathrm{ng}$ of pY005 plasmid template harboring wild type $P f d h f r, 10 \mu \mathrm{M}$ of sense primer F1 (CGGTGGATCCATGATGGAACAAG; $\mathrm{BamHI}$ site is underlined), $10 \mu \mathrm{M}$ of antisense primer R1 (CTTTGTCATCATTCTTAAGAGGC; AflII site is underlined), $0.1 \mathrm{mM}$ dGTP, $0.1 \mathrm{mM}$ dATP, $0.5 \mathrm{mM}$ dCTP, $0.5 \mathrm{mM}$ dTTP, $1 \times$ Mutagenesis buffer [16] and 5 units of GoTaq ${ }^{\circledR}$ DNA polymerase (Promega). The thermocycle condition was: 1 cycle of $95^{\circ} \mathrm{C}$ for $3 \mathrm{~min}$, 30 cycles of $95^{\circ} \mathrm{C}$ for $1 \mathrm{~min}, 50^{\circ} \mathrm{C}$ for $1 \mathrm{~min}, 72^{\circ} \mathrm{C}$ for $1 \mathrm{~min}$, and final extension of $72^{\circ} \mathrm{C}$ for $5 \mathrm{~min}$. PCR products of random mutant Pfdhfr library of about $0.7 \mathrm{~kb}$ were cloned into the BamHI/AflII sites of pY005. The plasmids containing Pfdhfr mutation libraries were grown in Luria Bertani broth containing $100 \mu \mathrm{g} / \mathrm{ml}$ ampicillin in a $37^{\circ} \mathrm{C}$ incubator shaker for $12-16$ hours. Plasmids were extracted and purified using a Qiaprep Spin Miniprep kit (Qiagen). Extracted plasmids were precipitated by isopropanol and re-suspended in $10 \mu \mathrm{l}$ TE buffer (10 mM Tris, 1 mM EDTA pH 8.0) for use in transfection experiment.

\section{Determination of pyrimethamine sensitivity to parental and transgenic parasites}

The sensitivity of pyrimethamine to inhibit the parental PbGFP parasite or transgenic parasites expressing PfDHFR mutant was determined by the 4-day suppressive test [17]. Five groups of five BALB/c mice per group were infected intravenously (i.v.) with $1 \times 10^{7}$ parasitized 
erythrocytes and treated with different concentration of pyrimethamine by intraperitoneal (i.p.) injection four hours post infection. The control group was treated with $5 \%(\mathrm{v} / \mathrm{v}) \mathrm{DMSO}$ in PBS $\mathrm{pH}$ 4.0. The experimental groups were treated with different doses of pyrimethamine through the same route for another 3 days. Twenty-four hours after the last treatment (day 4), percentages of parasitaemia were determined by microscopic counting of Giemsa-stained smears from mouse tail blood.

\section{Statistical analysis}

All statistical analyses were carried out using SigmaPlot software version 11 (Systat Software Inc., USA). For calculation of the growth inhibitory curve, parasitaemia of the control group was set as $0 \%$ inhibition. The non-linear regression for sigmoidal dose-response (variable slope) was used to calculate the $50 \%, 90 \%$ or $95 \%$ effective dose $\left(\mathrm{ED}_{50}, \mathrm{ED}_{90}\right.$ or $\left.\mathrm{ED}_{95}\right)$ values.

\section{Transfection, selection and identification of Pfdhfr random mutant libraries}

In vitro culture of PbGFP and P. berghei transfection were performed as described [13]. Briefly, parasitized blood was collected from a donor animal and cultured overnight in culture media (RPMI 1640 medium containing $20 \%$ heat inactivated fetal calf serum, $50 \mathrm{IU} / \mathrm{mL}$ neomycin and $25 \mathrm{mM}$ Hepes). Schizont stage parasites were purified from the overnight culture by Nycodenz gradient centrifugation. The merozoites were transfected with the circular plasmid DNA harboring $P f d h f r$ mutant libraries using the standard Amaxa Nucleofector protocol [13] and re-infected into animals by i.v. injection. Twentyfour hours after transfection, $0.25 \mathrm{mg} / \mathrm{kg}$ of pyrimethamine was used to treat the infected mice by i.p. injection daily. Smears were taken to check parasitaemia and when positive, left to multiply, until parasite numbers were adequate (about 3\% parasitaemia) for genomic analysis. Tail blood was drawn from infected animals on alternate days until parasitaemia reached $8-10 \%$ and genomic DNA was extracted from whole blood using a genomic DNA Mini Kit (Geneaid). The genomic DNA obtained was transformed into E. coli $\mathrm{DH} 5 \alpha$ to recover the circular plasmid DNA containing Pfdhfr mutants. The transformed bacterial colonies were picked and extracted for plasmid DNA using a Qiaprep Spin Miniprep kit (Qiagen). The sequences of Pfdhfr mutants were obtained by DNA sequencing (Biodesign Sequencing Service, Thailand).

\section{Transfection, selection and cloning of transgenic} $P$. berghei parasite

Plasmid containing resistant mutant $P f d h f r{ }^{S 108 N}$ was completely digested with HindIII and KasI restriction enzymes to generate linear plasmid. The 5' and 3'UTRs of $P b d h f r$-ts served as homologous recombination sites for replacement of the endogenous Pbdhfr-ts on chromosome 7 with $P f d h f r^{S 108 N}$. In vitro culture of PbGFP and $P$. berghei transfection were performed as described [13]. Twenty-four hours after transfection, $0.25 \mathrm{mg} / \mathrm{kg}$ of pyrimethamine was used to treat the infected mice by i.p. injection daily until the drug resistant parasites appeared. The integrated transgenic mutant parasite clones were obtained by the limiting dilution method [18].

\section{PCR analysis of transgenic $P$. berghei parasite}

The correct integration of $P f d h f r^{S 108 N}$ sequence into the genome via the 5' and 3'UTRs of the Pbdhfr-ts locus was determined by PCR. A 4.0-kb DNA fragment spanning the endogenous 5'UTR Pbdhfr-ts sequence and the introduced Pfdhfr was indicative of an integration event and was amplified using primers F2 (TTGAGCTACATAACTTCCATACAT) and R1 (described above). A 3.0kb DNA fragment spanning the introduced $P f d h f r$ and the endogenous 3'UTR Pbdhfr-ts sequence, indicative of a 3 ' integration event was amplified using primers F1 (described above) and R2 (CGATCTACACCTCTTCAT).

\section{Expression profile of transgenic mutant parasite}

The transgenic parasites expressed PfDHFR ${ }^{S 108 N}$-TS under the control of 5' and 3'UTRs of Pbdhfr-ts. To determine whether this promoter efficiently drives $P f d h f r^{S 108 N_{-}}-t s$ mRNA expression, reverse-transcription PCR (RT-PCR) was performed. Total RNA was isolated from blood stage transgenic $P$. berghei parasites using Trizol reagent (Invitrogen). cDNA was generated and used as template for amplification with gene specific primers for the $P f d h f r$ transgene using primers F1 and R1 (described above). Controls included specific primers for $P b d h f r$ gene: PbDTF (GGGGGGGGCATATGGAAGACTTATCTGAAACATTCG) and PbDTR (GGACTAGTGTACTT CCTCATTTGG) and P. berghei alpha tubulin gene: PbatubulinF (GCATGCTGGGAGCTATTTTG) and PbatubulinR (GCTGGTTCAAATGCTGAGTTTG). RT-PCR was performed using the same PCR condition as described above.

\section{Results}

\section{Determination of pyrimethamine sensitivity in wild type} PbGFP parasites

Experimental mice infected with wild type PbGFP parasites were treated with different concentrations of pyrimethamine. As shown in Figure 1, in vivo $\mathrm{ED}_{50}$ and $\mathrm{ED}_{95}$ of pyrimethamine against PbGFP was $0.02 \mathrm{mg} / \mathrm{kg}$ and $0.25 \mathrm{mg} / \mathrm{kg}$, respectively. The $\mathrm{ED}_{95}$ concentration of pyrimethamine was then used for selection of transgenic resistant parasites in subsequent experiments. 


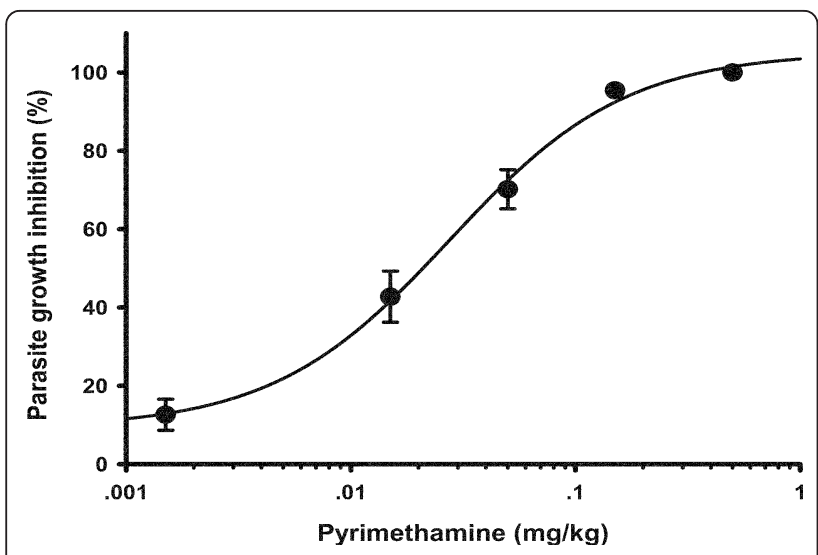

Figure 1 Sensitivity of pyrimethamine against PbGFP parasite. The data represents mean values \pm SD of percentage of growth inhibition for 5 animals per group. The average $\mathrm{ED}_{50}$ from three independent studies is $0.02 \mathrm{mg} / \mathrm{kg}$.

\section{Construction of Pfdhfr random mutant library using wild} type Pfdhfr template

Approximately 14,000 bacterial colonies were obtained for the Pfdhfr random mutant library. Twelve colonies were randomly picked and sequenced. From the sequence alignment, up to 4 base substitutions per gene were found. The mutation frequency was then calculated as $0.26 \%$, which is equivalent to approximately 2 base substitutions per 700 bp of Pfdhfr gene. Thus, the PCRinduced mutagenesis for this library is within the expected mutation frequency of 2 to 5 base substitutions per gene [19]. The rest of the bacterial colonies were harvested for library DNA preparation.

\section{Selection of transfected resistant mutant parasites from wild type Pfdhfr random libraries}

Plasmid DNA was purified from the Pfdhfr random mutant library and transfected to PbGFP parasites. Pyrimethamine-resistant parasites began to appear in most transfection experiments within 11 days post-transfection. Genomic DNA containing episomal transgenic DNA was extracted from drug-resistant parasites and transformed into $E$. coli. Among the isolated plasmids, two independent sequences were identified. One sequence contained a single base substitution mutation at amino acid position 108 (serine, S; AGC changed to asparagine, N; AAC) while the other had the same mutation at amino acid position 108 together with another base substitution at codon 196, which was silent (phenylalanine; TTT to TTㅡ).

\section{Generation of transgenic $P$. berghei expressing Pfdhfr $^{\text {S108N }}$-ts mutant}

In order to confirm that the $\mathrm{S} 108 \mathrm{~N}$ mutation found among library-transfected DNA from drug-resistant parasites conferred resistance, allelic replacement of the endogenous Pbdhfr-ts with mutant $P f d h f r^{S 108 N^{-}}$-ts was performed. The strategy for the allelic replacement event is shown in Figure 2. Correct integration was investigated by PCR analysis on genomic DNA using different primers pairs. PCR products obtained corresponded with the expected $4.0 \mathrm{~kb}$ and $3.0 \mathrm{~kb}$ bands for $5^{\prime}$ and 3 'UTR integration respectively, as shown in Figure 3A.

\section{Expression profile analysis of transgenic Pfdhfr ${ }^{S 108 N}$} mutant parasite

Transgenic $P$. berghei parasite with integrated Pfdhfr ${ }^{S 108 N_{-}}$-ts, named PbPfS108N, expresses the gene under the control of Pbdhfr-ts 5' and 3'UTRs. The expression of the enzyme was verified at the mRNA transcription level using RT-PCR. As shown in Figure 3B, 0.7 kb of $P f d h f r$ was amplified in cDNA from transgenic PbPfS108N parasite, but not in cDNA from PbGFP parental parasite. Conversely, Pbdhfr was amplified only in cDNA from parental PbGFP parasite, but not in cDNA from transgenic PbPfS108N parasites. The expected 0.85 $\mathrm{kb}$ band for the control P. berghei alpha tubulin was obtained for all parasites. No products were detected for -RT control templates, indicating that genomic DNA was absent.

\section{Determination of growth rate and pyrimethamine} sensitivity in transgenic $\mathrm{PbPfS108N}$ parasite

The growth rate of transgenic PbPfS108N parasite was compared with PbGFP parental parasite. The growth rates of the two parasites were not significantly different (Figure 4A). The efficacy of pyrimethamine to inhibit transgenic PbPfS108N parasites was determined by 4-day suppressive test. The calculated $\mathrm{ED}_{50}$ and $\mathrm{ED}_{90}$ values of

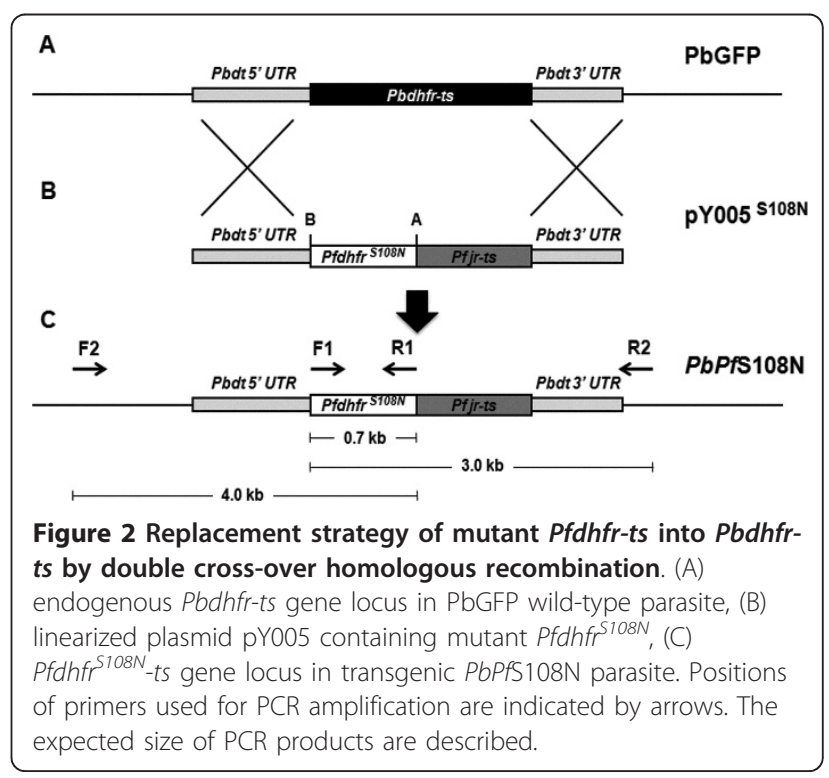




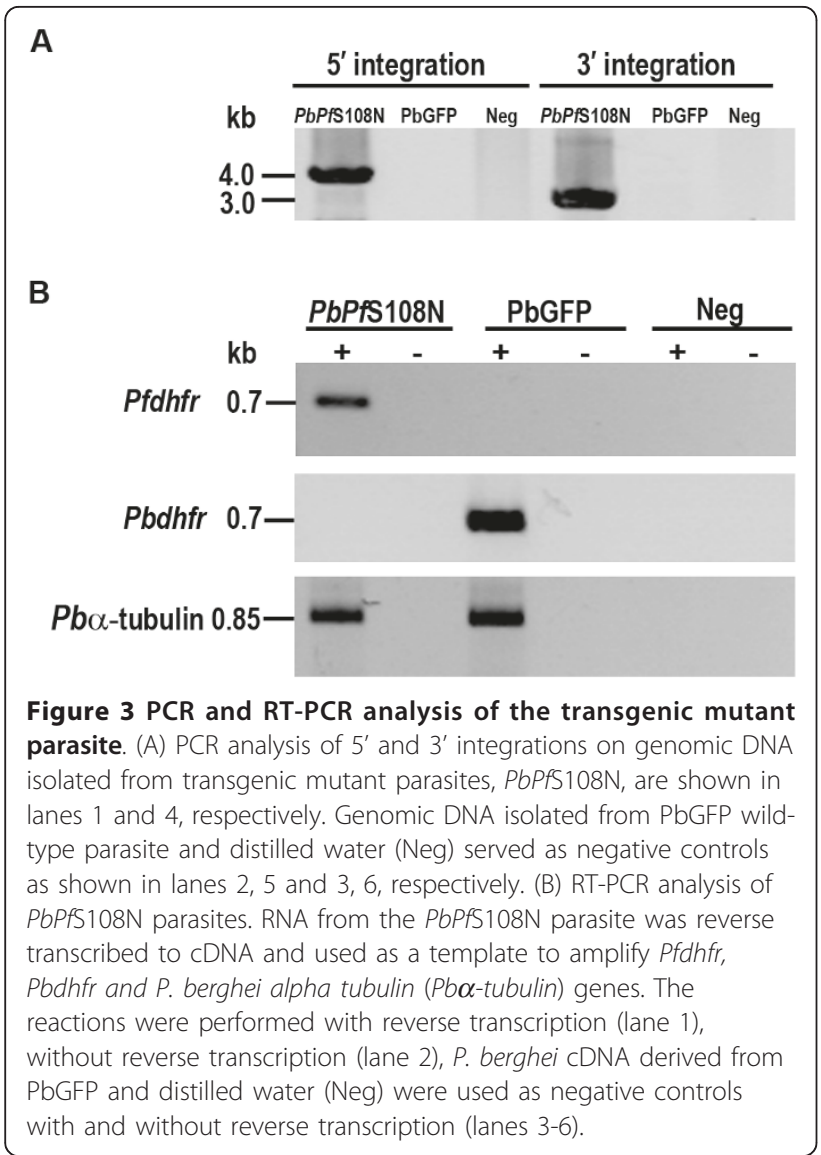

pyrimethamine against transgenic $P b P f S 108 \mathrm{~N}$ parasites were $1.33 \mathrm{mg} / \mathrm{kg}$ and $2.65 \mathrm{mg} / \mathrm{kg}$, respectively (Figure 4B).

\section{Discussion}

There is need to identify possible drug-resistance mutations against new compounds under development as antimalarial drugs. Prior knowledge of possible resistance pathways facilitates testing of strategies for forestalling the evolution and spread of resistance in the parasite population, e.g. drug combinations. In this study, a P. berghei in vivo model was developed as a surrogate cell expression system to identify Pfdhfr mutations that confer antifolate drug resistance. This system employs DNA transfection in which the selectable marker is antifolate-resistant mutant Pfdhfr, rather than the conventional human dhfr and Toxoplasma gondii dhfr markers used in P. berghei transfection [20]. The concentration of pyrimethamine at $0.25 \mathrm{mg} / \mathrm{kg}$ (ED ${ }_{95}$ of wild type $P$. berghei parasite) i.p. injection daily was successful in selecting resistant parasites obtained after transfection with Pfdhfr mutant libraries. Interestingly, the resistant parasite lines transfected with a $P f d h f r$ variant library were found to contain the S108N mutation, which is known to be the key antifolate resistance mutation

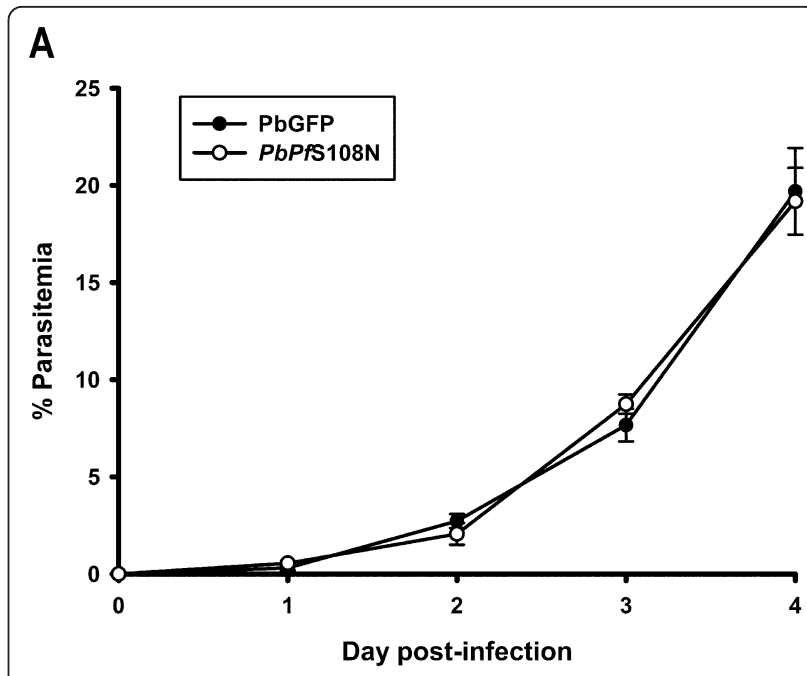

B

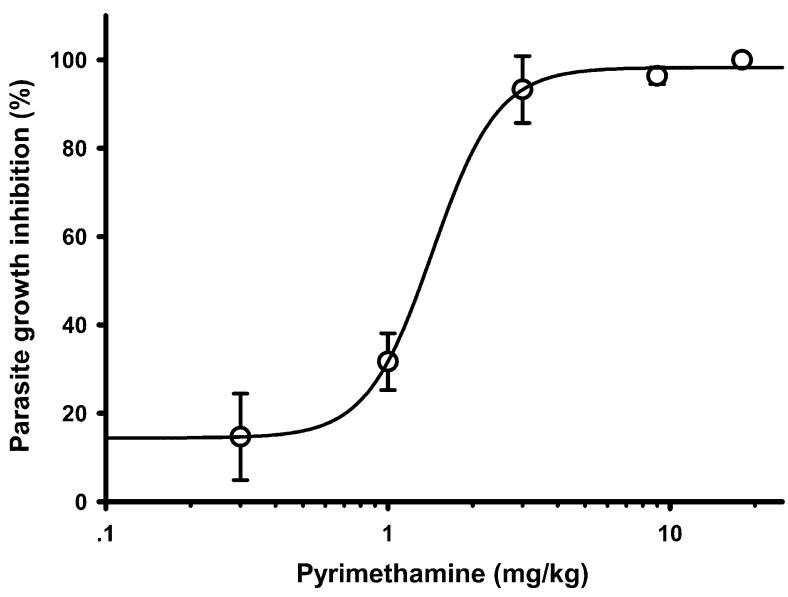

Figure 4 Growth rate and pyrimethamine sensitivity of PbPfS108N parasite. (A) Growth curves of PbPfS108N and PbGFP in mice. PbPFS108N data are represented as open circles and, PbGFP data represented as filled circles. The experiments were performed in three independent studies and the data represents mean \pm SD values. (B) Sensitivity of pyrimethamine against PbPfS108N parasite. The data represents mean \pm SD of percentage of growth inhibition. The average $\mathrm{ED}_{50}$ from three independent studies is $1.1 \mathrm{mg} / \mathrm{kg}$.

found in nature [21]. The fact that the pyrimethamine resistant line carried this mutation provides proof of concept of this system to be able to identify drug resistant mutations from a mutant library.

PfDHFR-TS crystal structure reveals the asparagine side chain of the mutant enzyme is in steric clash with the $p$ chlorophenyl moiety of pyrimethamine, which reduces the binding affinity of pyrimethamine for the mutant enzyme [10]. Although the selected drug-resistant library mutants contained the expressed S108N mutation, it should be noted that the drug-resistant library mutants are maintained episomally, and the increased $d h f r$ gene copy number in episomes may contribute to drug resistance in 
transgenic $P$. berghei, as has been shown for the $h d h f r$ transfection marker [20]. Thus, to test the hypothesis that pyrimethamine resistance in transgenic $P$. berghei is determined solely by the S108N mutant allele, Pbdhfr-ts was replaced with the Pfdhfr $S 108 N_{-}$-ts mutant by double homologous recombination. The integrated transgenic parasites expressed PfDHFR ${ }^{S 108 N}$-TS with a single copy of mutant $P f d h f r^{S 108 N}$ under the control of endogenous Pbdhfr-ts 5' and 3'UTRs. The resulting PbPfS108N transgenic parasites showed susceptibility to pyrimethamine with $\mathrm{ED}_{50}$ values 66-fold higher than PbGFP wild type, which strongly suggest that resistance is conferred by the S108N mutation. In addition, PbPfS108N transgenic parasites grew the same as PbGFP parasites. This result demonstrated that the DHFR-TS function is conserved between the two Plasmodium species, in agreement with other cross-species comparisons in the same genus [22]. Furthermore, the pyrimethamine resistance mutation had no negative effect on the function of the enzyme, in agreement with earlier studies [21,23]. This demonstrates the capacity of the transgenic $P$. berghei parasite model to study the fitness of Pfdhfr mutant alleles in the whole transmission cycle.

For pyrimethamine resistance, in addition to S108N mutation, other mutations such as N51I, C59R and I164L are known to augment the resistance level in parasites $[21,23]$. Although our mutant library is of sufficient diversity to contain these other mutations, they were not found among drug-selected parasites. In order to obtain transgenic parasites with a higher level of resistance, higher drug pressure will needed. However, selection of highly resistant pyrimethamine alleles with two or more mutations is not feasible, since mice could not tolerate the very high pyrimethamine doses needed.

In conclusion, a new experimental system for predicting the evolutionary pathway of antifolate drug resistance was developed. The major advantage of this system is that drug-resistant mutant alleles can be selected from diverse Pfdhfr libraries in a Plasmodium surrogate cell in a proper host background. Proof of concept for the system was demonstrated using the well-known antifolate pyrimethamine; however, new antifolates are being developed which are also effective against pyrimethamine-resistant parasites [24]. Therefore, the system could be utilized for identification of possible novel drug-resistant mutants that could arise against new antifolate compounds. This information could be used for rational design of effective anti-malarial drugs that forestall the emergence of drug resistance. Furthermore, our approach could also be applied to other Plasmodium enzyme drug targets for prediction the evolution of resistance mutations.

\section{Acknowledgements}

This work is supported by the UNICEF/UNDP/World Bank/WHO Special Programme for Research and Training in Tropical Diseases (TDR) to CU, the
Howard Hughes Medical Institute (HHMI, USA) to SK and the Faculty of Medicine, Chiang Mai University, Thailand to SS and WT. SK is an international research scholar of HHMI. WT was in receipt of Thailand Graduate Institute of Science and Technology (TGIST) studentship. We are grateful to Drs. Andrew Waters and Chris Janse for the provision of the transfection plasmid, transgenic $P$. berghei parasite line MRA-867 and their technical advice on $P$. berghei transfection. We thank Dr. Philip Shaw for his critical reading of the manuscript, Dr. Mofolusho Falade, Dr. Deanpen Japrung, Mr. Olugbenga Akinola for their technical support.

\section{Author details}

${ }^{1}$ National Center for Genetic Engineering and Biotechnology (BIOTEC), National Science and Technology Development Agency (NSTDA), 113 Thailand Science Park, Pathumthani 12120, Thailand. ${ }^{2}$ Department of Biochemistry, Faculty of Medicine, Chiang Mai University, Chiang Mai 50200, Thailand

\section{Authors' contributions}

CU, SS, SK and YY conceived and designed the project. WT performed all experiments. WT and CU prepared the manuscript. SS, SK and YY critically reviewed the manuscript. All authors read and approved the final manuscript.

\section{Competing interests}

The authors declare that they have no competing interests.

Received: 11 February 2011 Accepted: 10 May 2011

Published: 10 May 2011

\section{References}

1. World Health Organization: World malaria report 2009. Geneva, Switzerland: WHO Press; 2008.

2. Yuthavong $Y$ : Basis for antifolate action and resistance in malaria. Microbes Infect 2002, 4:175-182.

3. Cowman AF, Morry MJ, Biggs BA, Cross GA, Foote SJ: Amino acid changes linked to pyrimethamine resistance in the dihydrofolate reductasethymidylate synthase gene of Plasmodium falciparum. Proc Natl Acad Sci USA 1988, 85:9109-9113.

4. Sirawaraporn W, Prapunwattana P, Sirawaraporn R, Yuthavong Y, Santi DV: The dihydrofolate reductase domain of Plasmodium falciparum thymidylate synthase-dihydrofolate reductase. Gene synthesis, expression, and anti-folate-resistant mutants. J Biol Chem 1993, 268:21637-21644.

5. Peterson DS, Walliker D, Wellems TE: Evidence that a point mutation in dihydrofolate reductase-thymidylate synthase confers resistance to pyrimethamine in falciparum malaria. Proc Natl Acad Sci USA 1988, 85:9114-9118.

6. Hyde JE: The dihydrofolate reductase-thymidylate synthase gene in the drug resistance of malaria parasites. Pharmacol Ther 1990, 48:45-59.

7. Wu Y, Kirkman LA, Wellems TE: Transformation of Plasmodium falciparum malaria parasites by homologous integration of plasmids that confer resistance to pyrimethamine. Proc Natl Acad Sci USA 1996, 93:1130-1134

8. Ferlan JT, Mookherjee S, Okezie IN, Fulgence L, Sibley CH: Mutagenesis of dihydrofolate reductase from Plasmodium falciparum: analysis in Saccharomyces cerevisiae of triple mutant alleles resistant to pyrimethamine or WR99210. Mol Biochem Parasitol 2001, 113:139-150.

9. Bunyarataphan S, Leartsakulpanich U, Taweechai S, Tarnchompoo B, Kamchonwongpaisan S, Yuthavong Y: Evaluation of the activities of pyrimethamine analogs against Plasmodium vivax and Plasmodium falciparum dihydrofolate reductase-thymidylate synthase using in vitro enzyme inhibition and bacterial complementation assays. Antimicrob Agents Chemother 2006, 50:3631-3637.

10. Yuvaniyama J, Chitnumsub P, Kamchonwongpaisan S, Vanichtanankul J, Sirawaraporn W, Taylor P, Walkinshaw MD, Yuthavong Y: Insights into antifolate resistance from malarial DHFR-TS structures. Nat Struct Biol 2003, 10:357-365.

11. Chusacultanachai $S$, Thiensathit P, Tarnchompoo B, Sirawaraporn W, Yuthavong Y: Novel antifolate resistant mutations of Plasmodium falciparum dihydrofolate reductase selected in Escherichia coli. Mol Biochem Parasitol 2002, 120:61-72. 
12. de Koning-Ward TF, Janse CJ, Waters AP: The development of genetic tools for dissecting the biology of malaria parasites. Annu Rev Microbiol 2000, 54:157-185.

13. Janse CJ, Ramesar J, Waters AP: High-efficiency transfection and drug selection of genetically transformed blood stages of the rodent malaria parasite Plasmodium berghei. Nat Protoc 2006, 1:346-356.

14. Janse CJ, Franke-Fayard B, Waters AP: Selection by flow-sorting of genetically transformed, GFP-expressing blood stages of the rodent malaria parasite, Plasmodium berghei. Nat Protoc 2006, 1:614-623.

15. Franke-Fayard B, Trueman H, Ramesar J, Mendoza J, van der Keur M, van der Linden R, Sinden RE, Waters AP, Janse CJ: A Plasmodium berghei reference line that constitutively expresses GFP at a high level throughout the complete life cycle. Mol Biochem Parasitol 2004, 137:23-33.

16. Giver L, Gershenson A, Freskgard PO, Arnold FH: Directed evolution of a thermostable esterase. Proc Natl Acad Sci USA 1998, 95:12809-12813.

17. Peters $W$, Portus JH, Robinson BL: The chemotherapy of rodent malaria, $X X I I$. The value of drug-resistant strains of $P$. berghei in screening for blood schizontocidal activity. Ann Trop Med Parasitol 1975, 67:155-71.

18. Waters AP, Thomas AW, van Dijk MR, Janse CJ: Transfection of malaria parasites. Methods 1997, 13:134-147.

19. Cirino PC, Mayer KM, Umeno D: Generating mutant libraries using errorprone PCR. Methods Mol Biol 2003, 231:3-9.

20. de Koning-Ward TF, Fidock DA, Thathy V, Menard R, van Spaendonk RM, Waters AP, Janse CJ: The selectable marker human dihydrofolate reductase enables sequential genetic manipulation of the Plasmodium berghei genome. Mol Biochem Parasitol 2000, 106:199-212.

21. Sirawaraporn W, Sathitkul T, Sirawaraporn R, Yuthavong Y, Santi DV: Antifolate-resistant mutants of Plasmodium falciparum dihydrofolate reductase. Proc Natl Acad Sci USA 1997, 94:1124-1129.

22. Chanama M, Chanama S, Shaw PJ, Chitnumsub P, Leartsakulpanich U, Yuthavong $Y$ : Formation of catalytically active cross-species heterodimers of thymidylate synthase from Plasmodium falciparum and Plasmodium vivax. Mol Biol Rep 2010, 38:1029-1037.

23. Lozovsky ER, Chookajorn T, Brown KM, Imwong M, Shaw PJ, Kamchonwongpaisan S, Neafsey DE, Weinreich DM, Hartl DL: Stepwise acquisition of pyrimethamine resistance in the malaria parasite. Proc Natl Acad Sci USA 2009, 106:12025-12030.

24. Yuthavong Y, Kamchonwongpaisan S, Leartsakulpanich U, Chitnumsub P: Folate metabolism as a source of molecular targets for antimalarials. Future Microbiol 2006, 1:113-125.

doi:10.1186/1475-2875-10-119

Cite this article as: Tipsuwan et al: Selection of drug resistant mutants from random library of Plasmodium falciparum dihydrofolate reductase in Plasmodium berghei model. Malaria Journal 2011 10:119.

\section{Submit your next manuscript to BioMed Central and take full advantage of:}

- Convenient online submission

- Thorough peer review

- No space constraints or color figure charges

- Immediate publication on acceptance

- Inclusion in PubMed, CAS, Scopus and Google Scholar

- Research which is freely available for redistribution

Submit your manuscript at www.biomedcentral.com/submit
Biomed Central 